\title{
FDAAA legislation is working, but methodological flaws undermine the reliability of clinical trials: a cross-sectional study
}

Douglas H Marin dos Santos, Álvaro N Atallah

The relationship between clinical research and the pharmaceutical industry has placed clinical trials in jeopardy. According to the medical literature, more than $70 \%$ of clinical trials are industry-funded. Many of these trials remain unpublished or have methodological flaws that distort their results. In 2007, it was signed into law the Food and Drug Administration Amendments Act (FDAAA), aiming to provide publicly access to a broad range of biomedical information to be made available on the platform ClinicalTrials (available at https://www.clinicaltrials.gov). We accessed ClinicalTrials.gov and evaluated the compliance of researchers and sponsors with the FDAAA. Our sample comprised 243 protocols of clinical trials of biological monoclonal antibodies (mAb) adalimumab, bevacizumab, infliximab, rituximab, and trastuzumab. We demonstrate that the new legislation has positively affected transparency patterns in clinical research, through a significant increase in publication and online reporting rates after the enactment of the law. Poorly designed trials, however, remain a challenge to be overcome, due to a high prevalence of methodological flaws. These flaws affect the quality of clinical information available, breaching ethical duties of sponsors and researchers, as well as the human right to health. 
2 Douglas H. Marin dos Santos, PhD, Federal University of São Paulo (Unifesp), São Paulo, Brazil

3 Álvaro Nagib Atallah, PhD, Full Professor at Federal University of São Paulo (Unifesp), São 4 Paulo, Brazil

5 Corresponding author: Douglas H. Marin dos Santos, Rua Borges Lagoa, 564, Conjunto 63, 6 Ed. Espaço São Paulo, Vila Clementino, CEP 04038-000, São Paulo, SP, Brazil, Phone 5561

7 81183592, douglas.santos@agu.gov.br

\section{BACKGROUND AND INTRODUCTION}

Medical treatment, as a general rule, must rely on the best available clinical evidence. The strength of a therapeutic recommendation is a complex process, but usually systematic reviews of high quality randomized controlled trials are accepted as the gold standard (Gülmezoglu \&Villar, 2003).

Broad and free access to biomedical research is therefore essential to public health and individual clinical decisions. However, many researchers and sponsors of clinical trials have been negligent in disclosing their findings, impairing transparency of biomedical research.

Lack of transparency in clinical research has many different faces. However, it usually emerges in two respects. First, researchers and sponsors hide results from the public by failing to report or publish their findings or by publishing partial or fraudulent scientific papers. These behaviors are usually called selective publication of clinical trials ${ }^{(1)}$.

Second, many clinical trials have methodological flaws that stop them from being a fair and ethical test on whether the tested therapy is working (Goldcare, 2014). Poor methodological designs allow results to be manipulated in ways that distort benefits and risks, according to the purposes of sponsors or researchers. They will provide neither reliable results nor valid conclusions (Jadad et al., 1996). Such biased trials are a mere sham of evidence, unable to determine whether the assessed intervention is effective, efficient, or safe.

Selective publication and poorly designed trials can lead to tragic outcomes. Doctors and patients are misled, and policymakers are misinformed, resulting in ungrounded clinical and policy decisions. Risks of new drugs may be underestimated, efficacy may be overestimated, and the risk-benefit ratio can be changed, resulting in potentially life-threatening decisions and disastrous policy options (Turner et al., 2008). Such practices may endanger the right to health and undermine evidence-based medicine, breaching the ethical duties of researchers and sponsors (Gøtzsche, 2012; Every-Palmer \& Howick, 2014).

Lack of transparency affects a large proportion of clinical trials, both ongoing and completed, especially industry-funded trials (Bekelman, 2003). According to Gøtzsche (2012), 9 out of the 10 largest pharmaceutical companies were sued and signed corporate integrity agreements under civil and criminal law, due to unethical and unlawful practices in the United States. Between 1987 and 2010, the American government recovered more than US\$18 billion as a result of frauds associated with healthcare cases (US Dep. of Justice, 2010). Considering the U.S. budget for healthcare (Carter \& Cox, 2011) authorities believe that up to $8 \%$ is lost due to fraudulent practices (Grassley, 2011).

Abuses in biomedical research, however, cannot persist due to the potential harm to research participants, patients, and the population as a whole. The nexus between transparency, information, and the right to health is clear from the individual's perspective and is within the scope of public health. Information enables individuals to promote their own health, claim for quality services and adequate policies, control and follow the progressive realization of their rights, and consent freely about their own bodies and health (Neto, 2004). Adequate information 
also enables policymakers to build evidence-based guidelines, managing public health and its scarce resources on an optimal scale.

It means that any patient, health professional, researcher, and policymaker, individually or organized in groups, have the right to access information on available medicines and therapies, including the effectiveness, side effects, and risks. Researchers, sponsors, and governments, therefore, have the power and duty to comprehensively and accurately make health information publicly available (MacNaughton \& Hunt, 2006; Lemmens \& Telfer, 2012; World Health Organization, 2015).

Many efforts have been made to accomplish broader trial transparency. In 1997, the Food and Drug Administration Modernization Act (FDAMA, Section 113) launched the ClinicalTrials.gov ${ }^{(2)}$ website and required the registration ${ }^{(3)}$ of protocols of clinical trials ${ }^{(4)}$. In 2004, the International Committee of Medical Journal Editors (ICMJE) required trial registration on ClinicalTrials.gov in order to consider manuscripts for publication in any of its member journals. In 2008, the revised Declaration of Helsinki (DoH) stated that every clinical trial must be registered before recruitment of the first subject. These attempts - among others - were not enforced by penalties. Thus, although registration was enhanced, it was not as broad and comprehensive as policymakers and authorities expected.

In 2007, the U.S. Congress enacted the FDA Amendments Act (FDAAA) in order to expand the clinical trial registry database created by FDAMA. The FDAAA requires the registration of every protocol of clinical trials, other than phase 1, of any drug, biologic, or device that meets the legal definition of an applicable clinical trial ${ }^{(5)}$. FDAAA Section 801 also requires mandatory reporting of clinical trial results on website ClinicalTrials.gov (http://clinicaltrials.gov) ${ }^{(6)}$ not later than 1 year after the primary completion date ${ }^{(7)}$. The penalty for non-compliance is US\$10,000/day ${ }^{(8)}$. Both registration and results reporting must be achieved through the Protocol Registration System (PRS) of ClinicalTrials.gov (http://prsinfo.clinicaltrials.gov) ${ }^{(9)}$.

Although the FDAAA was the first legislation enforced by monetary penalties, the literature claims it has not been effective in reaching broader public access to clinical information (Law, Kawasumi \& Morgan, 2011; Kuehn, 2012; Gill, 2012). Prayle, Hurley \& Smyth (2012), in a study similar to ours, found that only $22 \%$ (163/738) of clinical trials registered on ClinicalTrials.gov reported results within the legal time frame. Nevertheless, the FDA did not acknowledge Prayle, Hurley \& Smyth's findings, suggesting that methodological flaws have biased the reliability of their research (Hawkes, 2012).

Thus, little is known regarding the methodological quality of clinical trials registered at ClinicalTrial.gov, the effectiveness of FDAAA 801, and its correlation with selective publication of clinical trials. Therefore, in order to contribute to the current literature, we decided to evaluate the patterns of transparency and the methodological quality of clinical trials registered on ClinicalTrials.gov. Due to its growing economic and therapeutic importance, we decided to assess only protocols of clinical trials of biological medical products $(10,11,12)$. In particular, we evaluated the top five global best selling monoclonal antibodies ( $\mathrm{mAb}$ ) adalimumab, bevacizumab, rituximab, trastuzumab, and infliximab.

We also examined the methodological quality of these studies in order to evaluate whether industry-funded trials have a poorer design, biasing the findings. Additionally, we evaluated the 
91 impact of FDAAA 801 on researchers' decisions to report results on ClinicalTrials.gov and 92 publish their findings in scientific medical journals.

93

94

95

96

97

98

99

100

101

102

103

104

105

106

107

108

109

110

111

112

113

114

115

116

117

118

119

120

121

122

123

124

125

126

127

128

129

130

131

132

133

134

135

136

\section{METHODS}

We performed an analytical cross-sectional study with data collection on the ClinicalTrials.gov website and on the databases PubMed, Embase, Lilacs, Cochrane Central, and Google Scholar.

On ClinicalTrials.gov, we searched for registered protocols of clinical trials on adalimumab, bevacizumab, rituximab, trastuzumab, and infliximab (see our search strategy and exclusion criteria in Appendix 1).

Based on the data gathered on ClinicalTrials.gov, in order to find whether completed trials were published, we searched for corresponding papers in journals indexed by PubMed, Embase, Lilacs, Cochrane Central, and Google Scholar (see our search strategy in Appendix 2).

We then extracted the relevant data from both protocols and published papers.

\subsection{Outcomes}

In order to establish the current patterns of selective publication of clinical trials, we assessed the proportion of published and unpublished trials and then associated our findings to the type of funding for each study. We also evaluated the proportion of positive, negative, partially positive, neutral, or inconclusive results among published papers.

In addition, we assessed the proportion of trials that have reported results on ClinicalTrials.gov and then associated our findings with the type of funding for each protocol.

In order to establish the methodological quality of the protocols, we assessed the proportion of single arm studies (13), "placebo-controlled" studies ${ }^{(14)}$ and "usual therapycontrolled" studies, ${ }^{(15)}$ and their correlation to the type of funding. Furthermore, we evaluated the type of masking used (16) (if any), the randomization of participants (if any), and the control groups (if any). At this point, we used the Jadad scale (Jadad et al., 1996) to evaluate the methodological quality of the clinical trials in our sample. We acknowledge the Jadad scale has some limitations ${ }^{(17)}$, and we don't ignore there are several other scales and checklists for quality assessment, such as the Delphi list (Verhagen et al., 1998), the CONSORT 2010 statement (Schulz, Altman \& Moher, 2010), and the Cochrane Collaboration's tool (Higgins et al., 2011). Nevertheless, we chose the Jadad scale because it is reliable, validated, and easy to use and understand, even for those who do not have specific training on clinical trials assessment (Sjögren \& Halling, 2002; Olivo et al., 2008).

Finally, so as to determine the effectiveness of FDAAA 801, we divided the original sample ( $n=243$ ) into three different subgroups: (a) studies completed before the enactment of FDAAA 801, (b) studies completed after FDAAA 801, but not covered by mandatory reporting, and (c) studies completed after FDAAA 801 and covered by mandatory reporting. We then assessed the proportion of published trials and reported results in each subgroup.

\section{RESULTS}

According to the search strategies described in Appendix 1, we found 442 protocols of clinical trials registered on ClinicalTrials.gov of the biologics adalimumab, bevacizumab, rituximab, trastuzumab, and infliximab. We excluded 199 protocols according to our exclusion criteria $^{(18)}$ (see also Appendix 1). There were 243 protocols remaining: adalimumab $(n=67)$, infliximab $(n=65)$, rituximab $(n=54)$, bevacizumab $(n=43)$, and trastuzumab $(n=14)$. 


\subsection{Publication of clinical trials, reporting of clinical trial results on ClinicalTrials.gov, and funding sources of clinical trials}

Regarding our sample of 243 protocols, we compared the proportion of published and unpublished studies. Through December 31, 2013, 178 clinical trials were published ( $\approx 73.3 \%)$ while 65 remained unpublished $(\approx 26.7 \%)$. The proportion of published papers for each biologic were as follows: adalimumab ( $\mathrm{n}=54 / 67 ; \approx 80.6 \%$ ), infliximab $(\mathrm{n}=50 / 65 ; \approx 77 \%$ ), rituximab $(\mathrm{n}=36 / 54 ; \approx 66.6 \%)$, bevacizumab $(\mathrm{n}=29 / 43 ; \approx 67.4 \%)$ and trastuzumab $(\mathrm{n}=10 / 14 ; \approx 71.4 \%)$.

With regard to reporting results on ClinicalTrials.gov, we found that only 73 trials $(\approx 30 \%)$ reported results online, as required by FDAAA 801 . By cross-referencing our findings, we also identified 38 trials $(\approx 15.6 \%)$ that were neither published nor reported at ClinicalTrials.gov. In that situation, data on clinical trials is entirely absent, in a way that it is not possible to assert whether the tested biologic works properly, if it is cost-effective, safe, and adequate for the condition of the patient.

Regarding the type of funding, we found $169(\approx 70 \%)$ industry-funded trials and $74(\approx 30 \%)$ independently funded trials (studies not funded by the pharmaceutical industry). Among unpublished clinical trials $(\mathrm{n}=65), 44(\approx 67.7 \%)$ were industry-funded while $21(\approx 32.3 \%)$ were independently funded.

\subsection{Does FDAAA 801 work? Possible impacts of U.S. legislation on subgroups S1, S2, and S3}

According to FDAAA 801, reporting results on ClinicalTrials.gov is mandatory up to 12 months after the completion of the study. Regarding our sample of 243 studies, only $73(\approx 30 \%)$ reported results online. Nevertheless, FDAAA 801 does not cover all trials registered on ClinicalTrials.gov, but only applicable clinical trials ${ }^{(19)}$.

Therefore, in order to find whether FDAAA 801 has positively affected publication and reporting rates of the clinical trials assessed, we divided our original sample into three different subgroups. The first subgroup (S1) comprised trials completed before the FDAAA (2002 through 2006). The second subgroup (S2) comprised trials completed after the FDAAA (2008 through 2012), but not covered by mandatory reporting, and the third subgroup (S3) comprised trials completed after the FDAAA (2008 through 2012) and under mandatory reporting (Fig. 1).

Regarding this specific outcome, we excluded all trials completed prior to 2002 and after 2012. We also excluded trials for which the completion date or, alternatively, the primary completion date, was not available on ClinicalTrials.gov $(n=55)$.

Figure 1.

In subgroup $1(\mathrm{n}=44), 28$ trials $(\approx 63.6 \%)$ were published and 6 trials $(\approx 13.6 \%)$ reported results on ClinicalTrials.gov. In subgroup $2(\mathrm{n}=87), 61$ trials $(\approx 70.1 \%)$ were published and 31 trials $(\approx 35.6 \%)$ reported results on ClinicalTrials.gov. Finally, in subgroup $3(\mathrm{n}=57), 48$ trials $(\approx 84.2 \%)$ were published and 40 trials $(\approx 70.2 \%)$ reported results on ClinicalTrials.gov.

When we compared subgroup 1 (trials completed prior to FDAAA enactment) with subgroup 3 (trials under mandatory reporting), the proportion of published studies significantly increased from $\approx 63.6 \%$ to $\approx 84.2 \%(\mathrm{p}=0.032)$ and the proportion of reported results rose from $\approx 13.6 \%$ to $\approx 70.2 \%(\mathrm{p}<0.001)$ (Tables 1 and 2$)$.

On the other hand, when we compared subgroup 2 (trials not under mandatory reporting) with subgroup 3 (trials under mandatory reporting), the proportion of published papers ( $\approx 70.1 \%$ 
183

versus $\approx 84.2 \%, \mathrm{p}=0.084)$ and reported results $(\approx 35.6 \%$ versus $\approx 70.2 \%, \mathrm{p}<0.001)$ was also increased (see Tables 1 and 2).

These findings suggest that FDAAA 801 may be positively influencing the proportion of published trials and reported results.

\section{Table 1.}

Table 2.

We also assessed the proportion of studies that were (a) both published and reported, (b) only published, (c) only reported, and (d) neither published nor reported. Our findings corroborate the above conclusion towards the effectiveness of FDAAA 801, as shown below in Table 3.

\section{Table 3.}

\subsection{Positive and negative results among published trials}

In order to find whether clinical trials with positive results are more likely to be published when compared with trials that have negative or neutral results as alleged by the literature (Rising et al., 2008), we screened our subsample of published trials $(\mathrm{n}=178)$ and evaluated each paper and its conclusions. That said, positive results were found in 118 papers $(\approx 66.3 \%)$, while negative results were described in 18 papers $(\approx 10 \%)$. Neutral or inconclusive results were reported in 11 trials $(\approx 7 \%)$, and partially positive findings were described in 24 trials $(\approx 13.5 \%)$. In 7 trials $(\approx 4 \%)$, the same biologic was tested using different dosages or different forms of administration.

\subsection{Substances assigned to the control group and their relation to funding sources}

According to the World Medical Association Declaration of Helsinki, the "benefits, risks, burdens and effectiveness of a new intervention must be tested against those of the best proven interventions". It means that within the context of an appropriately designed clinical trial, the new drug must be compared with a competitor that is known to be effective and safe (hereafter treatment as usual or TAU), in order to demonstrate the advantages or disadvantages of the new intervention ${ }^{(20)}$.

It is common, however, to compare the new intervention with a useless placebo substance, potentially distorting and biasing the results of the trial. It is also common, in worse scenarios, to find single arm studies (SAS), in which every participant enrolled receives the same experimental therapy.

Thus, in order to find the proportion of single arm studies, placebo-controlled, and TAUcontrolled trials in our sample ( $\mathrm{n}=243$ ), we assessed each protocol to determine the type of substance assigned as a control.

We found $84(\approx 35 \%)$ single arm trials, $53(\approx 22 \%)$ placebo-controlled trials, and $80(\approx 33 \%)$ TAU-controlled trials. We also found $13(\approx 5 \%)$ trials in which the new intervention was compared with placebo and TAU, and $13(\approx 5 \%)$ trials in which the intervention was tested using different dosages or administration forms. 
We then cross-referenced these findings with the type of funding for each clinical trial (industry-funded or independently funded) in order to find whether the source of funding affects, in any form, the design and reliability of the study (Table 4).

We found a higher prevalence of single arm studies $(62 / 169 ; \approx 36.7 \%$ versus $22 / 74$; $\approx 29.7 \%, \mathrm{p}=0,367)$ and placebo-controlled trials $(44 / 169 ; 26 \%$ versus $9 / 74 ; \approx 12.2 \%, \mathrm{p}=0,025)$ among industry-funded trials. On the other hand, we found TAU-controlled trials are more prevalent within independently funded trials when compared to industry-funded trials (36/74; $\approx 48.6 \%$ versus $44 / 169 ; \approx 26 \%, \mathrm{p}<0,001)$.

\section{Table 4.}

\subsection{Methodological design and quality of protocols}

Based on the information gathered on ClinicalTrials.gov, we evaluated the methodological quality of protocols. First, we assessed whether the trial was a single arm design (i.e. no comparison group) or group-designed (i.e. participants are allocated in different groups). Second, we examined whether the trial randomly allocated participants in groups (randomization). Finally, we examined whether the clinical trial was masked to treatment allocation (i.e. doubleblinded or single-blinded).

Out of the 243 protocols found, $159(\approx 65.4 \%)$ allocated participants into two or more control groups and $84(\approx 34.6 \%)$ were single arm trials. In addition, $149(\approx 61.3 \%)$ trials were randomized and $94(\approx 38.7 \%)$ were not randomized. Finally, $84(\approx 34.5 \%)$ trials were blinded while $159(\approx 65.4 \%)$ were not blinded.

Cross-referencing these findings, we determined that only 82 trials $(\approx 33.7 \%)$ were cumulatively group-designed, randomized, and masked, achieving a good or fair methodological design according to the Jadad scale (Jadad et al., 1996).

At this point, it is noteworthy that the monoclonal antibodies adalimumab, bevacizumab, rituximab, and trastuzumab received orphan drug designation for the treatment of some rare diseases, according to the Orphanet website (http://www.orpha.net). It is known that the quality of clinical trials of rare conditions may be impaired, with remarkable differences in design, blinding and randomization (Bell \& Smith, 2014). However, within our sample of 243 studies, only $11(\approx 4.5 \%)$ were associated with the rare diseases referred by Orphanet.

\section{DISCUSSION}

Our findings suggest that selective publication of clinical trials persists, regardless of the type of funding or intervention assessed (in our research, biologics). Through December 31, 2013 , about $25 \%$ of clinical trials were not published and $15 \%$ were not published and did not have results reported on ClinicalTrials.gov.

Online reporting of results on ClinicalTrials.gov also remains low, ranging between $7 \%$ (prior to the FDAAA) and $70.2 \%$ (trials covered by mandatory reporting). Nevertheless, we found a significant increase in publication and reporting rates after FDAAA 801. These findings suggest that the U.S. legislation is effective, achieving several of its goals.

Whereas the methodological quality of clinical trials is highly related to the transparency of clinical research, affecting its reliability and subsequent medical choices and health policies, we also assessed the methodological standards of registered studies. Not surprisingly, we found that approximately $67 \%(161 / 243)$ were graded as poor according to the Jadad scale. This means that 
273

274

275

276

277

278

279

280

281

282

283

284

285

286

287

288

289

290

291

292

293

294

295

296

297

298

299

300

301

302

303

304

305

306

307

308

309

310

311

312

313

314

315

316

317

only around one third of the protocols registered on ClinicalTrials.gov had a reliable methodological design (fair or good) (Jadad et al., 1996).

We also found that industry-funded trials are more likely to be single arm designed or placebo-controlled when compared to independently funded trials. On the other hand, TAUcontrolled trials were more common among independently funded trials.

These findings suggest that, despite the fact that industry has been reporting and publishing its trials in similar proportions to those of independent researchers, poor methodological choices may undermine the reliability of industry-funded trials.

Finally, we also assessed the prevalence of positive, negative, neutral, or inconclusive results among published trials $(\mathrm{n}=178)$. Positive $(\approx 66.3 \%)$ and partially positive $(\approx 13.5 \%)$ results were more prevalent, which is compatible with the literature (Decullier, 2005; Rising et al., 2008). These findings may result from the prevalence of studies with poor methodological quality in our sample. After all, $137(\approx 56.4 \%)$ trials were single arm designed or placebocontrolled trials and only 82 trials $(\approx 33.7 \%)$ were blinded and randomized. On the other hand, because our findings are solely quantitative, it is possible that the prevalence of positive results is associated with the true efficacy of the tested biologics.

Although our findings suggest that FDAAA 801 has had positive impacts on the dissemination and expansion of biomedical information and data, it is important to highlight that poorly designed trials remain as a major challenge for transparency. This is because it is not easy to determine whether a clinical trial has methodological flaws, particularly for nonprofessionals. A poorly designed trial can distort results in ways that drug benefits are overestimated and risks or harms are underestimated, unacceptably breaching ethical and moral duties of sponsors and researchers.

Indeed, a database that requires protocol registration and results submission, but does not separate the "wheat from the chaff", can potentially mislead health professionals, patients, and policymakers ${ }^{(21)}$. Therefore, legislation needs to go further in order to require researchers and sponsors to provide ClinicalTrials.gov with data on the quality of clinical trials.

Thus, beyond study registration and results submission, we believe researchers and sponsors should be legally required to self-rate their protocols, according to the Jadad scale or other assessment system, in order to inform patients, health professionals, and policymakers about the methodological quality of each trial made publicly available.

We assume future legislation must address the subject as a growing demand for human rights-based medicine in which health decisions are made in light of comprehensive information. Any legal initiative, however, is likely to become useless if a single core value is not universally shared. Otherwise, clinical research may become discriminatory, because the basic rights and duties of participants and researchers will be different according to where the study is performed. Discrepant legal systems may lead to human rights violations or unfair financial inducement (Terwindt, 2014).

A global agenda on transparency must be homogenous and standardized, enabling broad access to results, methodological quality, and funding sources of clinical trials. Providing reliable, comprehensive, and easy access to data on biomedical research, beyond safeguarding the human right to health and information, enables the expansion of systematic reviews and, as a consequence, evidence-based medical and health decisions ${ }^{(22)}$.

\subsection{Strengths and limitations of this study}


To our knowledge, this study is the first to evaluate the patterns of transparency of the clinical trials of biologics. We assessed different standards and trends associated with transparency: publication rates, reporting results on ClinicalTrials.gov, methodological flaws, and the impact of FDAAA 801 on clinical research. We also compared industry-sponsored trials with independently funded trials. Our findings set forth that, beyond reporting and publication bias, poor methodological quality of clinical trials is a challenge that must be faced in the near future.

Moreover, the methodology applied in our study was enhanced by the extensive search strategy for published papers employed on PubMed, Embase, Lilacs, Cochrane Central, and Google Scholar.

Finally, our research is aligned with recent World Health Organization Statement on Public Disclosure of Clinical Trial Results (2015). The WHO statement establishes that researchers must publicly report results in both of the following two modalities. First, "main findings of clinical trials are to be submitted for publication in a peer-reviewed journal within 12 months of study completion". Second, "key outcomes are to be made publicly available within 12 months of study completion by posting to the results section of the primary clinical trial registry" (World Health Organization, 2015). The available literature on the subject, to our knowledge, has primarily faced the patterns of results reporting at ClinicalTrials.gov (Zarin et al., 2011; Law, Kawasumi \& Morgan, 2011; Kuehn, 2012; Gill, 2012; Prayle, Hurley \& Smyth, 2012), which makes our study the first - or one of the first - to assess both reporting modalities currently recommended by WHO.

Our research, however, also has some limitations. First, we only assessed the protocols and publications of five biologics. Thus, because of sample bias, our findings may not represent the transparency patterns of clinical research as a whole. Indeed, the effectiveness of the FDAAA legislation may be limited to clinical trials of biologics.

In addition, we did not contact investigators (or other responsible parties) in order to confirm non-publication of their studies. We decided not to do so because contact details are not regularly disclosed on ClinicalTrials.gov. Furthermore, the literature suggests that investigators rarely answer questions about the publication of their trials (Stern \& Simes, 1997; Decullier, 2005; Ross et al., 2009).

Moreover, our findings may be partially biased due to incomplete or contradictory information posted on ClinicalTrials.gov (Chan, 2008; Ross et al., 2009; Smyth et al., 2011). However, we completed individual forms for each registered protocol that were manually checked for contradictions, potentially reducing bias risks.

It is important to highlight that our search strategy, even though based on the most significant available databases (PubMed, Embase, Cochrane Central, Lilacs, and Google Scholar), did not include any manual search of printed journals.

It is noteworthy that we had no information about studies that applied for exemptions from mandatory reporting on ClinicalTrials.gov. We also note that clinical trials of biologics previously approved by the FDA, but under investigation for new indications, are required to post results up to 2 years after completion. However, we were not able to identify these studies due to unavailable data at ClinicalTrials.gov.

Finally, we did not evaluate any other policies that could have influenced the outcomes assessed in this study. Nevertheless, we note that the FDAAA stands alone as the only legislation establishing monetary penalties for responsible parties who fail to comply with registration or results submission requirements. 


\subsection{Comparison with the literature}

Our findings are consistent with the literature. However, regarding the impacts of the FDAAA on reporting rates on ClinicalTrials.gov, it is noteworthy that Prayle, Hurley \& Smyth (2012), who used a similar methodology to this study, found significantly different results. According to their findings, only $22 \%$ of the results were posted online, while we found a significantly higher proportion of $70.2 \%$. The imbalance may be explained by the following reasons: (a) Prayle's sample was significantly larger and not limited to biologics; and (b) according to the FDA, Prayle's sample was biased, because they included protocols that did not meet the legal definition of an applicable clinical trial.

\section{CONCLUSION}

Patterns of selective publication of clinical trials of biologics do not differ from other major classes of medical products. Funding sources did not affect publishing and reporting rates, but industry-funded trials were more likely to have methodological flaws when compared to independently funded trials. Most of the trials were performed under poorly designed protocols, lowering the accuracy and biasing the risk-benefit analysis.

Reporting of results and publication rates of clinical trials of biologics were enhanced under FDAAA 801. Expanding similar legal regulations worldwide should be an indelible goal for the near future, establishing a new legal and policy framework for the right to health and information.

\section{REFERENCES}

Bekelman JE. 2003. Scope and Impact of Financial Conflicts of Interest in Biomedical Research: A Systematic Review. The Journal of the American Medical Association 289(4):454-465

Bell SA \& Smith CT. 2014. A comparison of interventional clinical trials in rare versus non-rare diseases: an analysis of ClinicalTrials.gov. Orphanet Journal of Rare Diseases 9:170

Berger VW \& Alperson SY. 2009. A General Framework for the Evaluation of Clinical Trial Quality. Reviews on Recent Clinical Trials 4(2): 79-88

Carter S \& Cox A. 2011. Obama's 2012 budget proposal: How $\$ 3.7$ trillion is spent. The New York Times. Available at http://www.nytimes.com/packages/html/newsgraphics/2011/0119budget (accessed 27 May 2014)

Chan AW. 2008. Bias, Spin, and Misreporting: Time for Full Access to Trial Protocols and Results. PLoS Medicine 5(11): e230

Decullier E. 2005. Fate of Biomedical Research Protocols and Publication Bias in France: Retrospective Cohort Study. British Medical Journal 331(7507):19

Dutta, AS. 2009. Discovery of New Medicines, in The Textbook of Pharmaceutical Medicine, 6th Edition (ed Griffin JP). Oxford: Wiley-Blackwell

Every-Palmer S \& Howick J. 2014. How evidence-based medicine is failing due to biased trials and selective publication. Journal of Evaluation in Clinical Practice 20(6):908-14

Gill CJ. 2012. How often do US-based human subjects research studies register on time, and how often do they post their results? A statistical analysis of the Clinicaltrials.gov database. BMJ Open 2:e001186-e01186 


\section{PeerJ Reviewing Manuscript}

407

408

409

410

411

412

413

414

415

416

417

418

419

420

421

422

423

424

425

426

427

428

429

430

431

432

433

434

435

436

437

438

439

440

441

442

443

444

445

Goldcare B. 2014. Bad Pharma: How Drug Companies Mislead Doctors and Harm Patients. New York: Faber \& Faber

Gotzsche PC. Big Pharma Often Commits Corporate Crime, and This Must Be Stopped. British Medical Journal 345:e8462

Grassley C. 2011. Grassley on Health Card Fraud Recovery, Legislative Plans. US Senate. Available at http://grassley.senate.gov/news/Article.cfm?customel_dataPageID_1502=30844 (accessed 13 June 2014)

Gülmezoglu AM \&Villar J. 2003. Up-to-date systematic reviews: the best strategy to select medical care: The WHO Reproductive Health Library No 6. The World Health Organization. Available at http://apps.who.int/rhl/Up-todate $\% 20$ systematic $\% 20$ reviews $\% 20$ the $\% 20$ best $\% 20$ strategy $\% 20$ to $\% 20$ select $\% 20$ medical $\% 20$ car e.pdf?ua $=1$ (accessed 13 July 2014)

Hawkes N. 2012. FDA Disagrees with BMJ Study That Found Clinical Trials Were Not Being Reported. British Medical Journal 344:e3277

Higgins JPT, Altman DG, Gøtzsche PC, Jüni P, Moher D, Oxman AD, Savović J, Schulz KF, Weeks L \& Sterne JAC. 2011. The Cochrane Collaboration's tool for assessing risk of bias in randomised trials. British Medical Journal 343:d5928

Jadad A, Moore RA, Carrol D, Reynolds DJ, Gavaghan DJ \& McQuay HJ. 1996. Assessing The Quality Of Reports Of Randomized Clinical Trials: Is Blinding Necessary? Controlled Clinical Trials 17(1):1-12

Law MR, Kawasumi Y \& Morgan SG. 2011. Despite law, fewer than one in eight completed studies of drugs and biologics are reported on time on ClinicalTrials.gov. Health Affairs 30:2338-2345

Kuehn BM. 2012. Few studies reporting results at US government clinical trials site. The Journal of the American Medical Association 307(7):651-653

Lemmens T \& Telfer C. 2012. Access to Information and the Right to Health: The Human Rights Case for Clinical Trials Transparency. American Journal of Law and Medicine 38:63-112

MacNaughton G \& Hunt P. 2006. Impact Assessments, Poverty and Human Rights: A Case Study Using the Right to the Highest Attainable Standard of Health. World Health Organization. Available at www.who.int/hhr/Series_6_ImpactAssessments_Hunt_MacNaughton1.pdf (accessed 10 December 2014)

Neto L. 2004. O Direito Fundamental à Disposição Sobre o Próprio Corpo: A Relevância da Vontade na Configuração do Seu Regime. Coimbra: Coimbra Editora

Olivo SA, Macedo LG, Gadotti IC, Fuentes J, Stanton T \& Magee DJ. 2008. Scales to assess the quality of randomized controlled trials: a systematic review. Physical Therapy Feb;88(2):156-75

Prayle AP, Hurley MN \& Smyth AR. 2012. Compliance with Mandatory Reporting of Clinical Trial Results on ClinicalTrials.gov: Cross Sectional Study. British Medical Journal 344: d7373

Regulations.gov. 2009. Human subject protection; foreign clinical studies not conducted under an investigational new drug application - notice of final rule. Available at 
446 http://www.regulations.gov/\#!documentDetail;D=FDA-2004-N-0061-0002;oldLink=false 447 (accessed 26 April 2015)

448 Reis C, Landim A \& Pieroni JP. 2011. Lições da experiência internacional e propostas para 449 incorporação da rota biotecnológica na indústria farmacêutica brasileira. BNDES Setorial 34: 5$450 \quad 44$

451 Rising K, Bacchetti P, Bero L \& Ioannidis J. 2008. Reporting Bias in Drug Trials Submitted to 452 the Food and Drug Administration: Review of Publication and Presentation. PLoS Medicine 453 5(11):e217

454 Ross JS, Mulvey GK, Hines EM, Nissen SE, Krumholz HM \& Sim I. 2009. Trial Publication 455 after Registration in ClinicalTrials.Gov: A Cross-Sectional Analysis. PLoS Medicine 456 6(9):e1000144

457 Schulz KF, Altman DG \& Moher D. 2010. CONSORT 2010 statement: updated guidelines for 458 reporting parallel group randomised trials. British Medical Journal 340:c332

459 Smyth RMD, Kirkham JJ, Jacoby A, Altman DG, Gamble C \& Williamson PR. 2011. Frequency 460 and Reasons for Outcome Reporting Bias in Clinical Trials: Interviews with Trialists. British 461 Medical Journal 342:c7153

462 Stern JM \& Simes RJ. 1997. Publication Bias: Evidence of Delayed Publication in a Cohort 463 Study of Clinical Research Projects. British Medical Journal 15:640-645

464 Sjögren P \& Halling A. 2002. Quality of reporting randomised clinical trials in dental and 465 medical research. British Dental Journal 192, 100-103

466 Terwindt C. 2014. Health rights litigation pushes for accountability in clinical trials in India.

467 Health and Human Rights Journal 11;16(2):e84-95

468 Turner EH, Matthews AM, Linardatos E, Tell RA \& Rosenthal R. 2008. Selective Publication 469 Of Antidepressant Trials And Its Influence On Apparent Efficacy. New England Journal of 470 Medicine 358:252-260

471 US Dep. of Justice. 2010. Fraud Statistics: Overview. Available at http://www.taf.org/FCA472 stats-2010.pdf (accessed 29 July 2014)

473 Verhagen AP, de Vet HC, de Bie RA, Kessels AG, Boers M, Bouter LM \& Knipschild PG. 474 1998. The Delphi list: a criteria list for quality assessment of randomized clinical trials for 475 conducting systematic reviews developed by Delphi consensus. Journal of Clinical 476 Epidemiology 51(12):1235-41

477 World Health Organization. 2015. WHO Statement on Public Disclosure of Clinical Trial 478 Results. Available at http://www.who.int/ictrp/results/reporting (accessed 27 April 2015)

479 Zarin DA, Tse T, Williams RJ, Califf RM \& Ide NC. 2011. The ClinicalTrials.gov Results 480 Database - Update and Key Issues. The New England Journal of Medicine 364:852-860

481

\section{ENDNOTES}

483 (1) Selective publication of clinical trials covers different behaviors. The literature suggests that 484 between $30 \%$ and $45 \%$ of registered clinical trials are never published (Turner et al., 2008; Ross 485 et al., 2009). Positive results are more likely to be published when compared to negative results 
486 (Decullier et al., 2005; Rising et al., 2008). Selective publication is derived from many causes, 487 including negligence of researchers, concerns about financial losses (Decullier et al., 2005; 488 Turner et al., 2008), lack of international legislation about transparency in clinical research, and 489 the complexity of peer-reviewed publication itself.

490 (2) "ClinicalTrials.gov is a Web-based resource that provides patients, their family members, 491 health care professionals, researchers, and the public with easy access to information on publicly 492 and privately supported clinical studies on a wide range of diseases and conditions. The Web site 493 is maintained by the National Library of Medicine (NLM) at the National Institutes of Health 494 (NIH). Information on ClinicalTrials.gov is provided and updated by the sponsor or principal investigator of the clinical study. Studies are generally submitted to the Web site (that is, registered) when they begin, and the information on the site is updated throughout the study. In some cases, results of the study are submitted after the study ends." Available at

498 http://clinicaltrials.gov/ct2/about-site/background (accessed 13 March 2014).

499 (3) According to the ClinicalTrials.gov website, registration is the "process of submitting and 500 updating summary information about a clinical study protocol from its beginning to end, to a 501 structured, Web-based registry that is accessible to the public, such as ClinicalTrials.gov."

502 Available at https://clinicaltrials.gov/ct2/about-studies/glossary (accessed 21 May 2014).

503 (4) According to the ClinicalTrials.gov website, a protocol is "the written description of a 504 clinical study. It includes the study's objectives, design, and methods. It may also include 505 relevant scientific background and statistical information." Available at

506 https://clinicaltrials.gov/ct2/about-studies/glossary (accessed 21 May 2014).

507 (5) According to the ClinicalTrials.gov website, registration "is required for trials that meet the 508 FDAAA 801 definition of an applicable clinical trial and were either initiated after September 509 27, 2007, or initiated on or before that date and were still ongoing as of December 26, 2007. 510 Trials that were ongoing as of September 27, 2007, and reached the Completion Date before 511 December 26, 2007, are excluded. (...) Applicable Clinical Trials generally include interventional 512 studies (with one or more arms) of FDA-regulated drugs, biological products, or devices that 513 meet one of the following conditions: the trial has one or more sites in the United States; the trial 514 is conducted under an FDA investigational new drug application or investigational device

515 exemption; [and] the trial involves a drug, biologic, or device that is manufactured in the United 516 States or its territories and is exported for research." Available at

517 https://clinicaltrials.gov/ct2/about-studies/glossary (accessed 21 May 2014).

518 (6) According to the ClinicalTrials.gov website, “(...) Responsible Parties must submit scientific 519 and administrative information about the results of the trial to the ClinicalTrials.gov results 520 database." Available at https://clinicaltrials.gov/ct2/manage-recs/how-report (accessed 05 May 521 2015).

522 (7) According to the ClinicalTrials.gov website, the primary completion date is the "date that the 523 last participant in a clinical study was examined or received an intervention and that data for the 524 primary outcome measure were collected. Whether the clinical study ended according to the 525 protocol or was terminated does not affect this date. (...) The primary completion date is the 526 term used in ClinicalTrials.gov for 'completion date' defined in Section 801 of the Food and 527 Drug Administration Amendments Act of 2007." Available at 528 https://clinicaltrials.gov/ct2/about-studies/glossary (accessed 22 May 2014). 
529 (8) According to the ClinicalTrials.gov website, the responsible party for a clinical trial is to be 530 penalized when the law is breached. The responsible party is the "sponsor, sponsor-investigator, 531 or sponsor-designated principal investigator who is responsible for submitting information about 532 a clinical study to ClinicalTrials.gov and updating that information." Available at 533 https://clinicaltrials.gov/ct2/about-studies/glossary (accessed 22 May 2014).

534 (9) According to the ClinicalTrials.gov website, researchers "must use ClinicalTrials.gov to 535 fulfill the requirements of FDAAA 801. FDAAA 801 requires Responsible Parties to submit 536 clinical trial information to the Director of the National Institutes of Health (NIH) for inclusion 537 in the registry and results database established via ClinicalTrials.gov". Available at 538 https://clinicaltrials.gov/ct2/manage-recs/faq\#complyFDAAA (accessed 03 May 2015).

539 (10) Biological products differ from drugs that are chemically synthesized in ways that affect 540 their cost, production, administration, and clinical efficacy (Morrow \& Felcone, 2004).

541 According to the FDA website, biologics "include a wide range of products such as vaccines, 542 blood and blood components, allergenics, somatic cells, gene therapy, tissues, and recombinant therapeutic proteins. Biologics can be composed of sugars, proteins, or nucleic acids or complex combinations of these substances, or may be living entities such as cells and tissues. Biologics are isolated from a variety of natural sources - human, animal, or microorganism - and may be produced by biotechnology methods and other cutting-edge technologies. Gene-based and cellular biologics, for example, often are at the forefront of biomedical research, and may be used to treat a variety of medical conditions for which no other treatments are available. (...) In contrast to most drugs that are chemically synthesized and their structure is known, most biologics are complex mixtures that are not easily identified or characterized. Biological products, including those manufactured by biotechnology, tend to be heat sensitive and susceptible to microbial contamination. Therefore, it is necessary to use aseptic principles from initial manufacturing steps, which is also in contrast to most conventional drugs. Biological products often represent the cutting-edge of biomedical research and, in time, may offer the most effective means to treat a variety of medical illnesses and conditions that presently have no other treatments available." Available at http:// http://www.fda.gov/AboutFDA/CentersOffices/OfficeofMedicalProductsandTobacco/CBER/uc m133077.htm (accessed 23 April 2015).

(11) By 2016, biologics will account for an estimated 21 percent share of the global pharmaceutical market. Monoclonal antibodies adalimumab, bevacizumab, rituximab, trastuzumab, and infliximab are among the top ten global best-selling medical products with 2016 estimated sales of US 37.8 billion dollars (Reis, Landim \& Pieroni, 2011).

(12) Monoclonal antibodies adalimumab, bevacizumab, rituximab, trastuzumab, and infliximab are used for the treatment of a wide range of diseases including breast cancer, pancreatic cancer, non small-cell lung cancer, metastatic colon or rectum cancer, non-Hodgkin's lymphoma, rheumatoid arthritis, psoriatic arthritis, plaque psoriasis, ankylosing spondylitis, Crohn's disease, and macular degeneration (Dutta, 2009).

(13) According to the ClinicalTrials.gov website, a single arm or a single group designed trial is described as "a clinical trial in which all participants receive the same intervention." Available at http://clinicaltrials.gov/ct2/about-studies/glossary (accessed 21 May 2014). 
574 (14) According to the ClinicalTrials.gov website, in a placebo-controlled trial, a "group of participants ... receives a placebo during a clinical study." Available at

576 http://clinicaltrials.gov/ct2/about-studies/glossary (accessed 21 May 2014).

577 (15) According to the ClinicalTrials.gov website, in a usual therapy-controlled study, a group of participants receives a comparison drug "that is considered to be effective." Available at http://clinicaltrials.gov/ct2/about-studies/glossary (accessed 21 May 2014). (16) According to the ClinicalTrials.gov website, masking (or blinding) is a clinical trial design strategy in which one or more parties involved with the trial, such as the investigator or participant, do not know which participants have been assigned which interventions. Available at http://clinicaltrials.gov/ct2/about-studies/glossary (accessed 21 May 2014).

(17) An adequate and comprehensive evaluation of clinical trials could assess up to ten different dimensions of the study. The Jadad scale rates only three of these dimensions, while Delphi rates six, Cochrane rates five, and the CONSORT report guide rates nine. Jadad scale, from this point of view, is less comprehensive and more subject to bias when compared to other similar tools (Berger \& Alperson, 2009).

(18) We excluded every clinical trial in which the assessed biologic was neither the primary intervention nor the primary comparator/control under evaluation (see Appendix 1 for detailed search strategy and exclusion criteria).

592 (19) Only trials that meet the definition of an applicable clinical trial are under the purview of

593 FDAAA 801. See note 5.

594 (20) The $6^{\text {th }}$ Revision of the Declaration of Helsinki states that the "benefits, risks, burdens and effectiveness of a new intervention must be tested against those of the best proven intervention(s)", except in cases where no proven intervention exists or where, for compelling methodological reasons, the use of placebo is necessary to determine the efficacy or safety of an intervention and the patients who receive placebo will not be subject to additional risks of serious or irreversible harm. Nevertheless, in October 2008 the FDA removed references to the $\mathrm{DoH}$, in reaction to the restrictions on the use of placebo-controlled trials. According to the FDA, the U.S. government "continues to support the Declaration's underlying principles. However, 602 (...) the U.S. Government does not fully support the 2000 version of the Declaration because it contains certain statements that may be inconsistent with U.S. law and policy (e.g., concerning use of placebos in clinical trials) (Regulations.gov, 2009). Thus, from the perspective of the FDA, the use of placebo within the assessed sample is not an ethical violation, while the $\mathrm{DoH}$ points in the exact opposite direction. While we acknowledge how controversial the subject is, it is noteworthy we found a significant higher prevalence of placebo-controlled studies among industry-funded trials, potentially revealing a link between economic interests and the use of placebo.

610 (21) Berger \& Alperson (2009) stress that "in many cases, flawed or misleading evidence is 611 worse than no evidence at all. This is because the state of ignorance resulting from a lack of 612 evidence is recognized as a state of ignorance, whereas the state of ignorance resulting from 613 misleading evidence is not so recognized. In addition, the existence of any clinical trials, 614 misleading or not, effectively precludes the possibility of planning future trials to address the 615 same questions as those addressed by the existing trials. For these reasons, misleading evidence 616 in the form of flawed clinical trials is quite troublesome to public health". 
1

Definition of subgroups $\mathrm{S} 1, \mathrm{~S} 2$, and $\mathrm{S} 3$

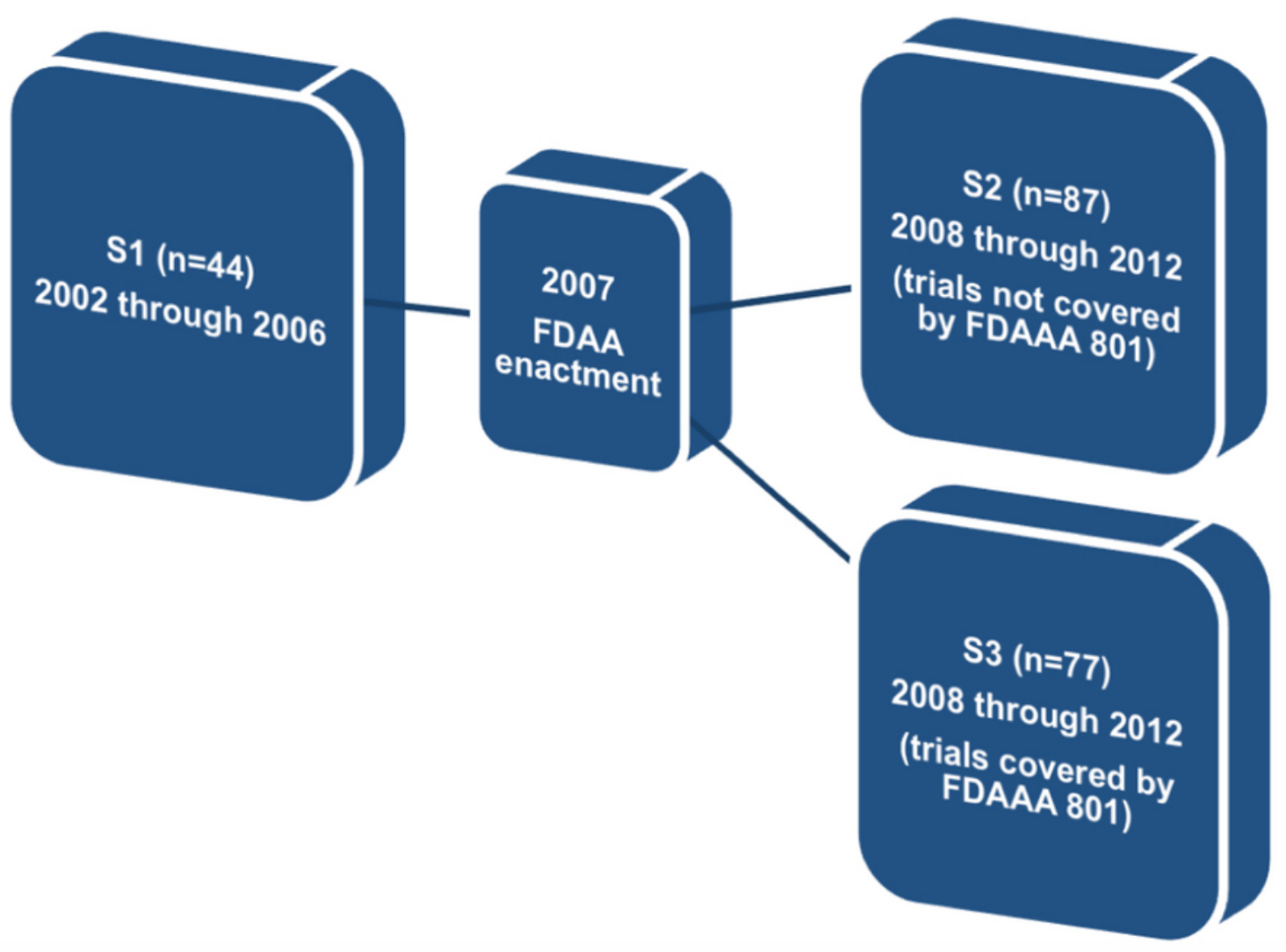




\section{Table $\mathbf{1}$ (on next page)}

Proportion of Reported and Unreported Results on ClinicalTrials.gov (Subgroups S1, S2, and $\mathrm{S} 3, \approx \%)$ 
2

\begin{tabular}{ccccccc}
\hline \multirow{2}{*}{ Subgroup } & \multicolumn{2}{c}{ Unreported } & \multicolumn{2}{c}{ Reported } & \multicolumn{2}{c}{ Total } \\
\cline { 2 - 7 } & $\mathrm{n}$ & $\mathbf{0}$ & $\mathrm{n}$ & $\%$ & $\mathrm{n}$ & $\%$ \\
\hline & & & & & & \\
S1 & 38 & 86.4 & 6 & 13.6 & 44 & 100 \\
S2 & 56 & 64.4 & 31 & 35.6 & 87 & 100 \\
S3 & 17 & 29.8 & 40 & 70.2 & 57 & 100 \\
& & & & & &
\end{tabular}

3 


\section{Table 2 (on next page)}

Proportion of Published and Unpublished Trials (Subgroups S1, S2, and S3, $\approx \%$ ) 
2

\begin{tabular}{ccccccc}
\hline & \multicolumn{2}{c}{ Unpublished } & \multicolumn{2}{c}{ Published } & \multicolumn{2}{c}{ Total } \\
\cline { 2 - 7 } Subgroup & $\mathrm{n}$ & $\%$ & $\mathrm{n}$ & $\%$ & $\mathrm{n}$ & $\%$ \\
\hline & & & & & & \\
S1 & 16 & 36.4 & 28 & 63.6 & 44 & 100 \\
S2 & 26 & 29.9 & 61 & 70.1 & 87 & 100 \\
S3 & 9 & 15.8 & 48 & 84.2 & 57 & 100 \\
& & & & & & \\
\hline
\end{tabular}

3 


\section{Table 3(on next page)}

Proportion of Clinical Trials: (a) Both Published and Reported, (b) Only Published, (c) Only Reported, and (d) Neither Published nor Reported (missing data) $(\approx \%)$ 


\begin{tabular}{|c|c|c|c|}
\hline & $\begin{array}{c}\text { Subgroup } 1 \\
\text { Pre-FDAAA } 801 \\
(n=44)\end{array}$ & $\begin{array}{l}\text { Subgroup } 2 \\
\text { Not Under } \\
\text { Mandatory } \\
\text { Reporting } \\
(n=87)\end{array}$ & $\begin{array}{c}\text { Subgroup } 3 \\
\text { Under } \\
\text { Mandatory } \\
\text { Reporting } \\
(n=57)\end{array}$ \\
\hline $\begin{array}{l}\text { (a) Trials both } \\
\text { published and } \\
\text { reported }\end{array}$ & $\begin{array}{c}\mathrm{n}=1 \\
(\approx 2.3 \%)\end{array}$ & $\begin{array}{c}\mathrm{n}=17 \\
(\approx 19.5 \%)\end{array}$ & $\begin{array}{c}\mathrm{n}=34 \\
(\approx 59.7 \%)\end{array}$ \\
\hline $\begin{array}{l}\text { (b) Studies } \\
\text { published only }\end{array}$ & $\begin{array}{c}\mathrm{n}=27 \\
(\approx 61.3 \%)\end{array}$ & $\begin{array}{c}n=44 \\
(\approx 50.5 \%)\end{array}$ & $\begin{array}{c}\mathrm{n}=14 \\
(\approx 24.5 \%)\end{array}$ \\
\hline $\begin{array}{l}\text { (c) Results reported } \\
\text { only }\end{array}$ & $\begin{array}{c}\mathrm{n}=5 \\
(\approx 11.4 \%)\end{array}$ & $\begin{array}{c}\mathrm{n}=14 \\
(\approx 16.1 \%)\end{array}$ & $\begin{array}{c}\mathrm{n}=6 \\
(\approx 10.5 \%)\end{array}$ \\
\hline (d) Missing data & $\begin{array}{l}n=11 \\
(25 \%)\end{array}$ & $\begin{array}{c}\mathrm{n}=12 \\
(\approx 13.8 \%)\end{array}$ & $\begin{array}{c}\mathrm{n}=3 \\
(\approx 5.2 \%)\end{array}$ \\
\hline Total & $\begin{array}{c}n=44 \\
(100 \%)\end{array}$ & $\begin{array}{c}n=87 \\
(100 \%)\end{array}$ & $\begin{array}{c}n=57 \\
(100 \%)\end{array}$ \\
\hline
\end{tabular}




\section{Table 4(on next page)}

Substances Assigned to the Control Group According to the Funding Sources of Each Trial (Industry-funded or Independently Funded) 
2

\begin{tabular}{lcccccc}
\hline & $\begin{array}{c}\text { TAU } \\
(n=80)\end{array}$ & $\begin{array}{c}\text { Placebo } \\
(n=53)\end{array}$ & $\begin{array}{c}\text { TAU and } \\
\text { Placebo } \\
(n=13)\end{array}$ & $\begin{array}{c}\text { Single } \\
\text { Arm } \\
(n=84)\end{array}$ & $\begin{array}{c}\text { Different Dosages } \\
\text { or Administration } \\
\text { Forms }(n=13)\end{array}$ & $\begin{array}{c}\text { Total } \\
(n=243)\end{array}$ \\
\hline $\begin{array}{l}\text { Industry- } \\
\text { funded }(\mathrm{n}=169)\end{array}$ & $\mathrm{n}=44$ & $\mathrm{n}=44$ & $\mathrm{n}=13$ & $\mathrm{n}=62$ & $\mathrm{n}=6$ & $\mathrm{n}=169$ \\
& $(\approx 26 \%)$ & $(\approx 26 \%)$ & $(\approx 7.7 \%)$ & $(\approx 36.7 \%)$ & $(\approx 3.5 \%)$ & $(100 \%)$ \\
$\begin{array}{l}\text { Independently } \\
\text { funded }(\mathrm{n}=74)\end{array}$ & $\mathrm{n}=36$ & $\mathrm{n}=9$ & -- & $\mathrm{n}=22$ & $\mathrm{n}=7$ & $\mathrm{n}=74$ \\
& $(48.6 \%)$ & $(\approx 12.2 \%)$ & & $(\approx 29.7)$ & $(\approx 9.5 \%)$ & $(100 \%)$ \\
\hline
\end{tabular}

3 